\title{
Experimental Assessment of 10Gbps 5G Multicarrier Waveforms for High-layer Split u-DWDM-PON-based Fronthaul
}

\author{
Samael Sarmiento, Jose Antonio Altabas, Salvatore Spadaro, Senior Member, IEEE, and Jose A. \\ Lazaro, Member, IEEE
}

\begin{abstract}
The current constant growth in mobile networks' traffic demands caused by the popularisation of cloud and streaming services on personal devices, requires architectural changes so as to fulfil all new $5 \mathrm{G}$ mobile network requirements. Cloud access radio network (C-RAN) architecture in combination with the massive deployment of small cell antenna sites have recently been proposed as a promising solution but will be demanding for high capacity mobile fronthaul links. An efficient way for performing that connectivity is to make use of the dense wavelength multiplexing passive optical network (DWDM-PON) infrastructure. In this context, OFDM has been extensively explored as a potential candidate. Nevertheless, the main drawback of OFDM is its high out-of-band radiation. In order to overcome that drawback, new 5G multicarrier waveforms (FBMC, UFMC and GFDM) have recently been proposed. In this paper, we experimentally assess and compare 10Gbps 32-QAMOFDM/FBMC/UFMC/GFDM system performance for high-layer split ultra-DWDM-PON-based fronthaul using a radio-over-fiber technique. The performance has been done in terms of spectral efficiency, peak-to-average power ratio, spectral density and receiver sensitivity. In particular, intensity-modulation with direct-detection and quasi-coherent-detection have been considered. In order to improve the multicarrier system energy efficiency, the effect of using a hard clipping technique over transmitted signals is also studied. Finally, we evaluated the crosstalk interference between two adjacent channels of the same modulation scheme, as a function of their electrical frequency span for downlink application.
\end{abstract}

Index Terms - Filter-bank based multi-carrier, fronthaul, generalised frequency division multiplexing, orthogonal frequency division multiplexing, radio-over-fiber, universal filtered multicarrier.

\section{I.INTRODUCTION}

$\mathrm{W}$ ith the current exponential growth of the use of cloud and new multimedia streaming services on personal devices, along with the deployment of the "Internet of Things" (IoT) and massive machine-to-machine communications [1]-[4], it is expected that modern macrocell wireless networks will reach

Manuscript received November 13, 2018; revised ??; accepted ??. This work was supported in part by ALLIANCE (TEC2017-90034-C2-2-R) and FOANT (TEC2017-85752-R) projects co-funded by FEDER, MINECO FPI-BES-2015074302 and MECD FPU-13/00620. their limits in terms of capacity, flexibility, reliability and latency very soon [5]. To overcome that situation, small-cellbased mobile networks are considered as the most promising solution. However, in that network solution, a large number of small cells must be deployed to provide wide coverage. This makes it particularly important to simplify remote cell sites so as to reduce cost and increase energy efficiency. In this context, cloud access radio networks (C-RANs) have recently been proposed as a hopeful network architecture solution [6], [7]. That solution considers the division of the traditional base station, which integrated all the functionalities of radio and baseband processing, into several only-transmitting simple and cheap remote radio heads (RRHs) and a single cloud-hub base band unit (BBU) located at the central office (CO), where complex processing is centralised. As a consequence of that, a new connectivity segment called "fronthaul" is deployed between the multiple distributed RRHs and the centralised BBU. On the one hand, the most common standard, used by fronthaul vendors, is known as common public radio interface (CPRI) [8]. That standard uses the digitalised baseband signal transmission over fiber (DBBoF) technique, where the base band IQ samples are digitised and serially transmitted at a constant bit rate. As a result, CPRI transmission needs huge bandwidth. For example, for $100 \mathrm{MHz}$ radio bandwidth and multiple-input multiple-output (MIMO) degree of 8 , the data rate per sector of the fronthaul transmission system should be of 49.3Gbps [9], [10]. To overcome this issue, alternatively, radio-over-fiber (RoF) technology has recently gained more and more attention [11]-[15], not only for simplifying the interfaces of both BBUs and RRHs (digitisation and format conversion are not required), but also for saving bandwidth. Thus, for the aforementioned scenario, the RoF-based fronthaul just needs $2.4 \mathrm{GHz}$ of bandwidth [15]. That means a reduction of the required bandwidth of 30 times regarding to CPRI when the OOK modulation format is used. On the other hand, massive small cell deployment requires a huge number of optical fibers. To resolve that issue, the high-layer split dense wavelength

S. Sarmiento, S. Spadaro and J. A. Lazaro are with the School of Telecommunications Engineering, Polytechnic University of Catalonia, 08034 Barcelona, Spain, e-mail: samael.sarmiento@tsc.upc.edu.

J. A. Altabas is with the Department of Electrical Engineering and Communications, Aragon Institute of Engineering Research, University of Zaragoza, 50018 Zaragoza, Spain. 


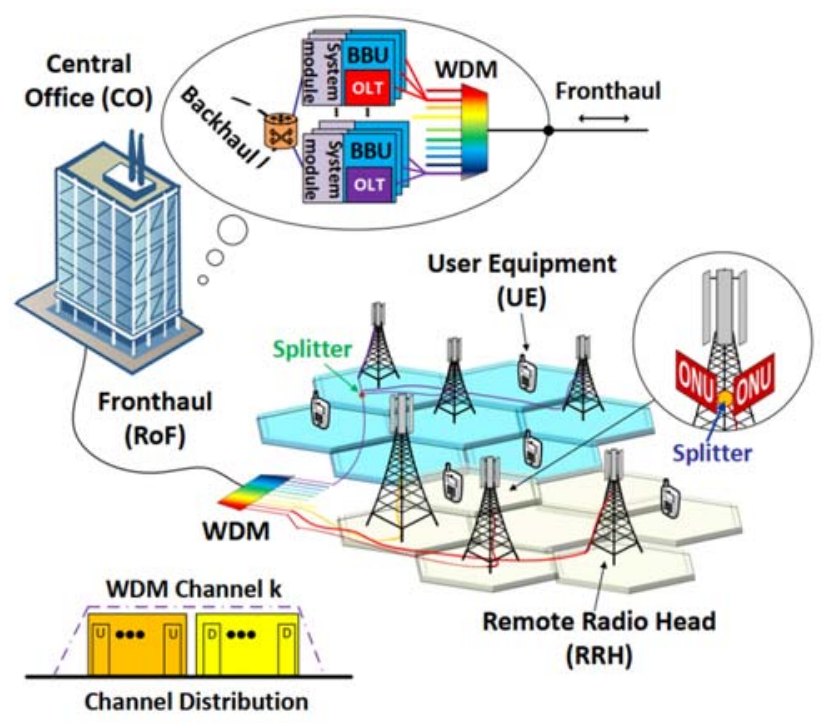

Fig. 1: High-layer split DWDM-PON-based fronthaul solution. Inset: Proposed flexible full-duplex DWDM division.

multiplexing passive optical network (DWDM-PON)-based fronthaul solution shown in Fig. 1 has been proposed in [16]. In this scenario, transmission techniques based on ultra-dense wavelength multiplexing (u-DWDM) are a promising alternative to time division multiplexing (TDM) solutions, due to their high spectral efficiency [17]. u-DWDM is accomplished by dividing each $100(200) \mathrm{GHz}$ WDM channel into two sub-channels, one for up-link and other for down-link, where different user demands can be allocated (see Fig. 1). In this way, all antennas connected to the same PON share the same DWDM channel. Benefits of this network architecture solution include the coexistence of different traffic natures and policies, low congestion at the access nodes, compatibility with legacy systems and industrial temperature ranges, and provides similar performances to standard dual fiber networks [18], [19].

In C-RANs, the design of the access to the medium is essential to improve the system capacity and to dynamically allocate the available resources. Orthogonal frequency division multiplexing (OFDM) [20]-[22] has been adopted in fourth generation $(4 \mathrm{G})$ networks, brought to the fact that it brings about numerous benefits, such as high resilience and efficient/flexible resource management. However, traditional OFDM is unable to accomplish all the new demands required for $5 \mathrm{G}$ networks, such as reduced out-of-band (OOB) radiation and, high power and spectral efficiency. In order to address these new challenges in 5G networks, filter-bank based multicarrier (FBMC), generalised frequency division multiplexing (GFDM) and universal filtered multicarrier (UFMC), all considered as new $5 \mathrm{G}$ orthogonal multicarrier waveforms, have recently been proposed as promising candidate technologies [23].

In this paper, we assume intensity-modulation (IM) with direct-detection (DD) and quasi-coherent detection (QCD) as being the most cost-effective solutions for high-layer split uDWDM-PON-based fronthaul implementation with RoF technology. In particular, we experimentally assess and compare 10Gbps 32-quadrature amplitude modulation (QAM)OFDM/FBMC/GFDM/UFMC system performances in terms of spectral efficiency, peak-to-average power ratio (PAPR), spectral density and receiver sensitivity. In order to improve multicarrier system energy efficiency, the effect of using the hard clipping technique over transmitted signals is also studied. Next, we evaluate the crosstalk interference between two adjacent channels of the same modulation scheme, as a function of their electrical frequency span for downlink application. Finally, the maximum fronthaul network split layer is discussed.

The remainder of this paper is organised as follows: Section II describes the experimental setup used for the multicarrier modulation schemes evaluation, Section III shows obtained results, and Section IV completes the paper with the main conclusions.

\section{II.EXPERIMENTAL SETUP}

The experimental setup is depicted in Fig. 2. The BBU's transmitter (BBU-TX) is based on an external cavity tunable laser source (TLS), modulated by a Mach-Zehnder Modulator (MZM). The MZM is biased at its quadrature point. The BBUTX uses a digital transmitter (DTX) where bits are randomly generated and coded into 32-QAM format. The obtained complex symbols are digitally modulated according to OFDM, UFMC, FBMC and GFDM modulation schemes [23]. In order to adapt the obtained complex signals to real ones that can be modulated and detected in amplitude, a juxtaposing technique in time domain is used [24]. This technique consists of splitting the complex output into real and imaginary parts and sending one after the other. The obtained real signal, oversampled to avoid aliasing issues, is optionally hard clipped and filtered so as to reduce the out-of-band clipping noise. A low-pass, finiteimpulse response (FIR) filter is considered.

The modulated signals are converted from digital-toanalogue using a 4GHz-bandwidth arbitrary waveform generator (AWG) set at $8 \mathrm{GSa} / \mathrm{s}$, obtaining the $2 \mathrm{GHz}$-bandwitdh signals shown in Fig. 2. To exhaust the MZM linear range, electrical amplification is used. The modulated optical signal is amplified to obtain $0 \mathrm{dBm}$ at BBU-TX's output by an erbiumdoped fiber optical amplifier (EDFA). The optical signal-tonoise ratio (OSNR) is then at $41 \mathrm{~dB}$. So, to reduce the amplified spontaneous emission noise over the optical signal, it is filtered using a $100 \mathrm{GHz}$ bandpass filter, i.e., emulating a WDM channel at $1545 \mathrm{~nm}$. The optical signal is launched into $25 \mathrm{~km}$ of standard single-mode fiber (SSMF). A second transmitter, with features similar to the described one, is added to the setup, so as to evaluate the crosstalk penalty between the two adjacent channels.

Two different cost-effective optical receivers are considered for the RRH implementation (see Fig. 2). The first one, a DD receiver, simply based on a PIN photodetector, combined with an electrical amplifier (EA), and the second one, a single ended QCD receiver based on the DD receiver. The local oscillator (LO) is a cost-effective distributed feedback laser (DFB). In the proposed setup, the LO and the signal are coupled at the photodiode using an optical coupler (DC) and a polarisation 


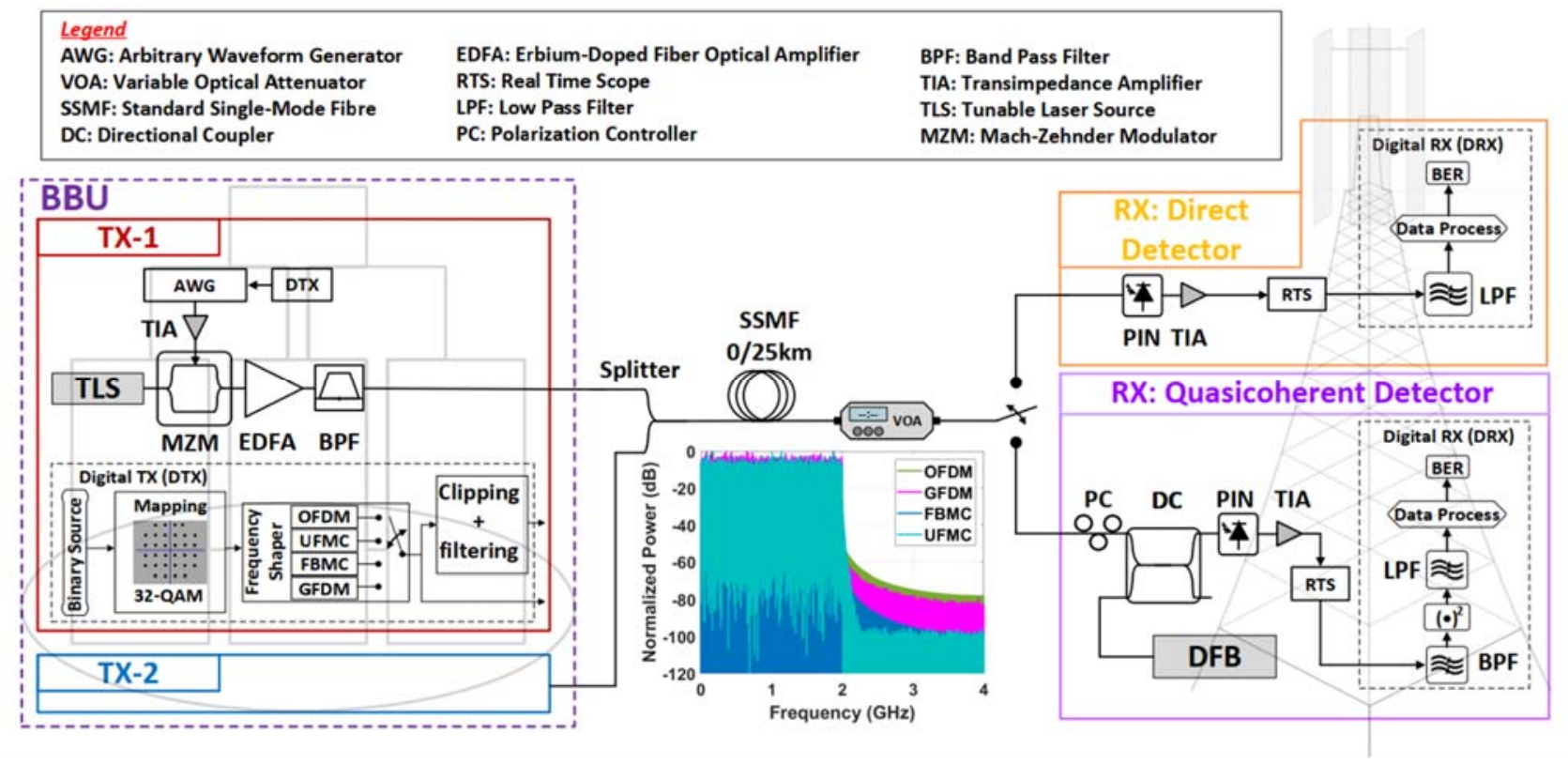

Fig. 2: Experimental setup. Inset: Measured electrical base-band spectra at the transmitter of 10Gbps 32-QAM-OFDM/GFDM/FBMC/UFMC before its optical conversion.

controller (PC). This QCD receiver can be easily upgraded to a polarisation insensible QCD receiver as indicated in [25], [26]. The bias current of the DFB is configured to provide $+14.3 \mathrm{dBm}$. After the heterodyne detector, the received signal is optically down-converted to an intermediate frequency (IF) equal to $5 \mathrm{GHz}$. For both proposed receivers, the detected signal is digitalised with a $25 \mathrm{GSa} / \mathrm{s}$ real time oscilloscope (MSO70804C) to be processed off-line.

Both DD and QCD receivers have a digital receiver (DRX) in which the first step is the low-pass and band-pass filtering of the digitalised signal with a FIR filter so as to reduce the noise, respectively. In the case of the QCD receiver, the band-pass filtered signal is multiplied with itself and low-pass filtered with a FIR filter as well. The filtered signals are now digitally demodulated according to the received signal scheme [23]. Finally, the bit-error rate (BER) is calculated, comparing the detected data stream to the original one.

\section{RESULTS}

In this section, three experimental studies have been done in order to evaluate and compare the performances of new $5 \mathrm{G}$ multicarrier waveforms and OFDM for u-DWDM-PON-based fronthaul implementation with RoF technology. The first study involves spectral efficiency, PAPR and power spectral density (PSD) measurements. The second study is focused on the sensitivity performance of considered multicarrier waveforms for the two proposed cost-effective receivers for the RRH implementation. The effects of clipping on the performance of the considered multicarrier waveforms, including PAPR, PSD and BER, have been investigated as well. For all considered multicarrier modulation schemes, we studied the spectral separation required between two adjacent channels of the same type, for a feasible BER degradation in downlink application. Finally, according to obtained results, the maximum fronthaul network split layer is discussed.

A. Spectral efficiency, PAPR and power spectral density comparison

New 5G multicarrier waveforms can be classified into two categories depending on the orthogonal modulation technique used [23]. While FBMC and GFDM use a pulse shaping technique, UFMC is based on sub-band filtering. FBMC can be understood as a modification of legacy OFDM, in which each sub-carrier is filtered to minimize its side-lobes, reducing, thus, the OOB noise of the global modulated signal. To maximize the OOB noise of this multicarrier waveform as much as possible, a PHYDYAS filter with an overlapping symbol factor, $K$, of 4 is considered [27]. Unlike FBMC, GFDM uses circular shifted filters to perform the pulse shaping over a data block. In GFDM, a data block consists of $N$ sub-carriers and $M$ time slots, transmitting $N \cdot M$ complex modulated data. In this case, a root raised cosine (RRC) filter with different roll-off factors, $\alpha$, is considered, defining $M=15$ for each GFDM block and 2 overlapped sub-carriers. Finally, UFMC considers different sub-bands, with equal size, and each sub-band is filtered with a shifted version of the same prototype filter. Specifically, a Dolph-Chebyshev filter with a length of 73, a side-lobe attenuation of $40 \mathrm{~dB}$ and different sub-band size, is contemplated. In all cases, it is considered $N=1024$ of which 512 are data sub-carriers. A cyclic prefix length, $N_{C P}$, of 72 samples is added to OFDM and GFDM. In the following, a broad set of experimental results that allows comparison of the spectral efficiency, the PAPR and the power spectral density of considered multicarrier waveforms is shown. For the sake of simplicity, they were done using the DD receiver, which is simply based on the PIN photodiode, plus the electrical amplifier (see Fig. 2). 


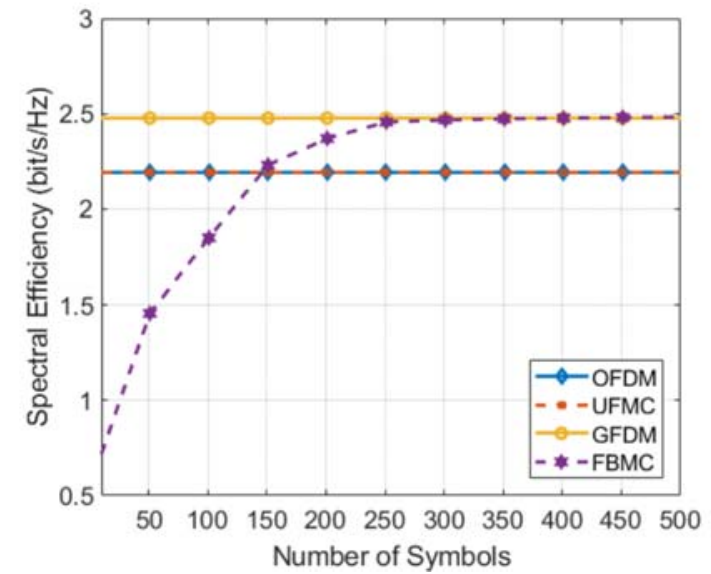

Fig. 3: Measure of spectral efficiency for different burst duration for OFDM, UFMC, GFDM and FBMC.

Figure 3 shows the spectral efficiency (SE) of OFDM, UFMC, GFDM and FBMC, in bits/s/Hz, for a frame with a different number of symbols. From Fig. 3, it can be deduced that for OFDM, UFMC and GFDM, the SE does not depend on the burst duration, while for FBMC it does so. In particular, the SE loss of FBMC for a low number of symbols is due to the transient state of the shaping filter. For a high number of symbols, the SE of FBMC is similar to GFDM. For GFDM, the SE is higher than OFDM; this is because the GFDM symbol is $M$ times longer than an OFDM symbol. Then, the SE relation between OFDM and GFDM is $\sim\left(1+N_{C P} / N\right)$ [28]. The UFMC SE is equal to OFDM since the length of the DolphChebyshev filter used is chosen for a fair comparison [28]. Finally, different values of $\alpha$ and sub-band size were considered for GFDM and UFMC, respectively. No significant changes were obtained in the SE.

PAPR, defined as $\left|x_{\text {peak }}\right|^{2} / x_{r m s}^{2}$, where $x$ represents the signal samples of a symbol, is a key performance parameter of a multicarrier communication system, since it has a direct bearing on the cost and energy efficiency of the hardware equipment expended. Moreover, any multicarrier signal with a high number of sub-carriers, as those under consideration here, $N=1024$, has high PAPR. Several alternative solutions to reduce the PAPR of transmitted signals have been proposed in the literature and most of them are overviewed in [29]. One of these approaches, and the simplest, is amplitude clipping of the multicarrier signal. Since high peaks take place in the transmission with very low probability, clipping could be an effective technique for PAPR reduction. Nevertheless, clipping is a nonlinear process that causes significant in-band distortion, degrading the bit-error-rate (BER) performance and increasing OOB noise. Filtering after clipping shrinks the OOB noise but also leads to some peak regrowth. Here, we studied, through extensive experimental measurements, the effects of clipping and filtering on the PAPR and PSD of OFDM, UFMC, FBMC and GFDM, for considered optical systems. By measuring the PAPR of the received signal and comparing the PAPR values of the generated signals before and after their transmission, it is

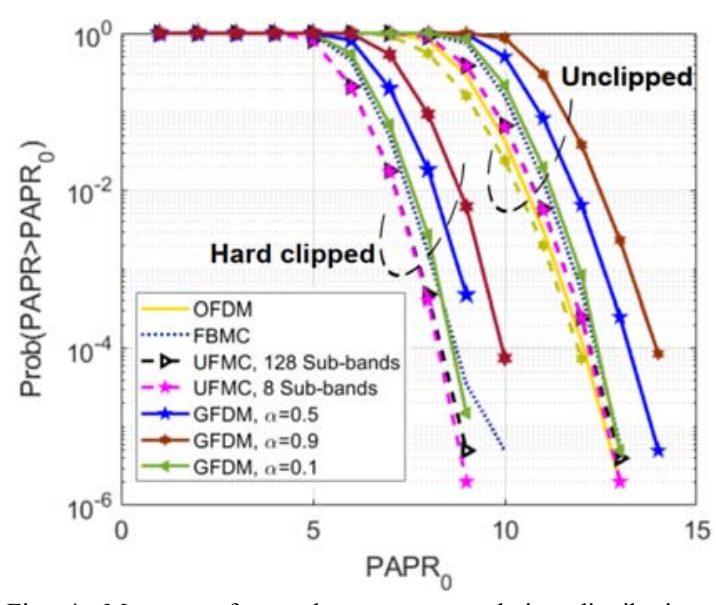

Fig. 4: Measure of complementary cumulative distribution function (CCDF) of PAPR for OFDM, FBMC, UFMC and GFDM through the optical system when the transmitted signals are unclipped and clipped.

possible to know if the complete system introduces nonlinear distortions over the transmitted signals. In all checked cases, no variation on the PAPR values was obtained, and, hence, the use of the clipping technique is justified to improve the energy efficiency of the communication system, as long as the BER degradation can be bearable, as it shall be studied in Section III.B.

Figure 4 shows the complementary cumulative distribution function (CCDF), defined as the probability that PAPR exceeds a certain value of $\mathrm{PAPR}_{0}$ vs. $\mathrm{PAPR}_{0}$, of OFDM, UFMC, FBMC and GFDM, measured at the receiver side, when the transmitted signal is unclipped and hard clipped to $50 \%$ of the peak. These results represent an average of $10^{6}$ realisations of a transmitted sequence through the system, each having the length of 10 symbols. For GFDM and UFMC signal generation, $\alpha=\{0.1,0.5$, $0.9\}$ and $N_{S B}$ (number of sub-bands) $=\{8,128\}$ have been considered, respectively. From Fig. 4, it can be observed that OFDM exhibits the best performance within the chosen parameters. However, it should also be noted that, for example, for a probability $(P)$ of $10^{-3}$, the gap between OFDM and the new $5 \mathrm{G}$ multicarrier waveforms is small, around $0.5 \mathrm{~dB}$ when $\alpha=0.1$ for GFDM. From Fig. 4 , the additional degree of freedom of GFDM to control the PAPR by just changing the roll-off factor of the raised cosine used as pulse shaper, can also be observed. Thus, varying $\alpha$ from 0.9 to 0.1 , a PAPR reduction of $1.5 \mathrm{~dB}$ can be achieved for $P=10^{-3}$. For UFMC, different subband sizes do not change the CCDF of the PAPR curves. Fig. 4 also shows the CCDF of PAPR for OFDM, FBMC, UFMC and GFDM when signals are clipped to $50 \%$ of the peak of each transmitted symbol. As it can be observed, the OFDM PAPR is reduced from $\sim 11.5 \mathrm{~dB}$ to $\sim 7.5 \mathrm{~dB}$ for $P=10^{-3}$. That is a reduction of $4 \mathrm{~dB}$. Similar PAPR reduction can be seen for FBMC, UFMC and GFDM.

In Fig. 5, the measured PSD of OFDM, FBMC, UFMC and GFDM signals when they are unclipped and hard clipped to $50 \%$ of the peak, is shown, in comparison with the electrical spectrum shown in Fig. 2, corresponding to an ideal wireless base-band signal, non-affected by optical modulator nonlinearity. The effect of clipping over the OOB noise is clear. 


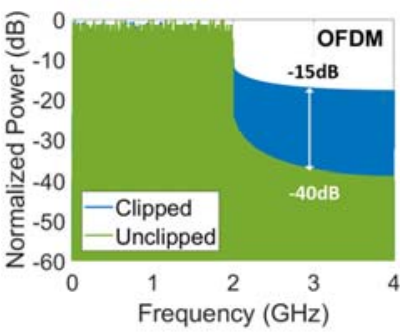

(a)

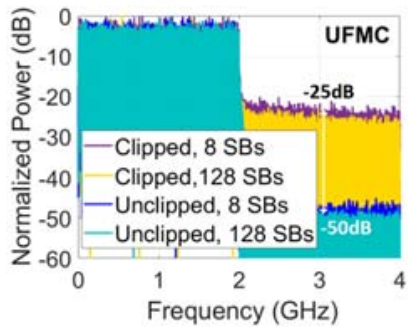

(c)

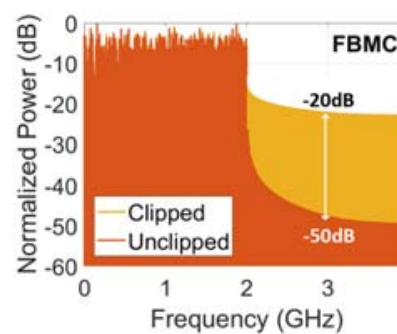

(b)

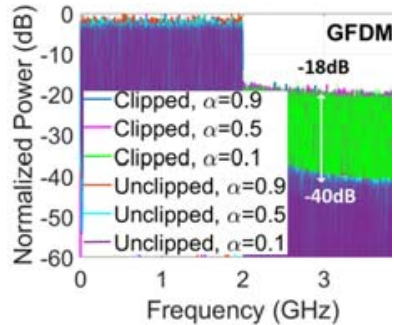

(d)

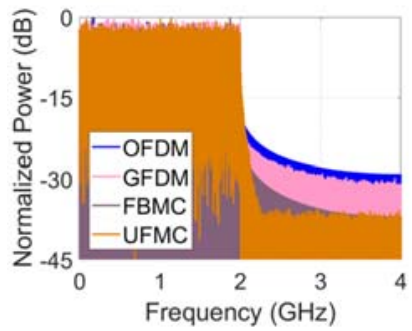

(e)

Fig. 5: Measure of power spectral density of: (a) OFDM, (b) FBMC, (c) UFMC and (d) GFDM signals when they are unclipped and clipped to $50 \%$ of the peak. (e) Comparison of measured power spectral density of OFDM, FBMC, UFMC and GFDM when signals are clipped and filtered.

Thus, for example, for a frequency of $3 \mathrm{GHz}$, the OOB noise power of hard clipped OFDM, see Fig. 5(a), is $-15 \mathrm{~dB}$ lower than the signal power, while for unclipped OFDM it is $-40 \mathrm{~dB}$. Similar OOB noise behaviour to OFDM can be observed for FBMC, UFMC and GFDM, when the signal is hard clipped, see Fig. 5(b)-(d). In these cases, the OOB noise level of FBMC, UFMC and GFDM are $-20 \mathrm{~dB}, \quad-25 \mathrm{~dB}$ and $-18 \mathrm{~dB}$ lower than the signal power, respectively. For GFDM and UFMC, changing the $\alpha$ and $N_{S B}$ values do not significantly impact on the OOB noise. All those results show that filtering is required to reduce the increase of the OOB noise level caused by clipping. Fig. 5(e) shows the PSD when OFDM, FBMC, UFMC and GFDM signals are hard clipped and filtered with a FIR filter. Now, for a considered frequency of $3 \mathrm{GHz}$, the difference between the power signal and OOB noise are around $-28 \mathrm{~dB}$, $30 \mathrm{~dB},-37 \mathrm{~dB}$ and $-37 \mathrm{~dB}$ for OFDM, GFDM, FBMC and UFMC, respectively. From Fig. 5(e), it can be noticed that UFMC offers the best spectral localisation followed by FBMC. GFDM has a little lower OOB noise level compared to OFDM.

\section{B. Sensitivity}

In this section, OFDM, FBMC, UFMC $\left(N_{S B}=128\right)$ and GFDM $(\alpha=0.1)$ performances have been measured in terms of the sensitivity for the two different cost-effective receivers proposed in Fig. 2. The first one consists of a simple DD receiver, and the second one is the single ended QCD receiver,

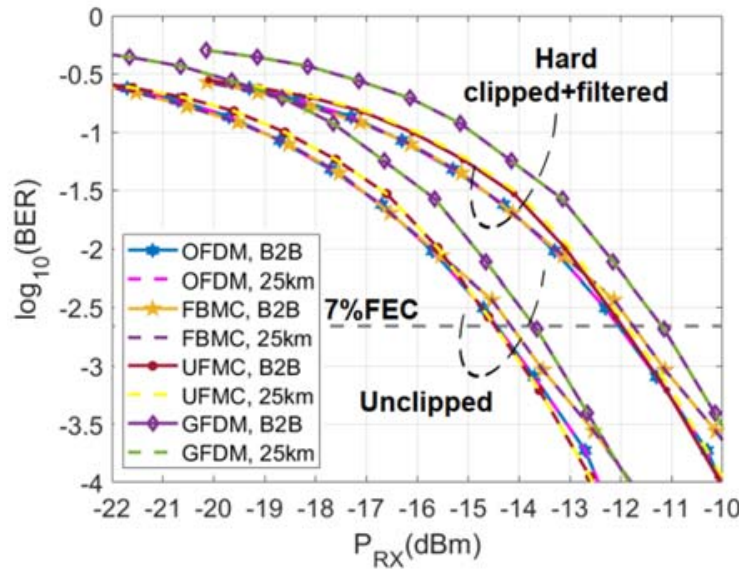

Fig. 6: BER vs. received optical power $\left(\mathrm{P}_{\mathrm{RX}}\right)$ of unclipped and clippedand-filtered OFDM, UFMC, GFDM and FBMC signals for DD receiver.

TABLE I

Summary of B2B sensitivities of unclipped and clipped-and-filtered OFDM, FBMC, UFMC and GFDM signals for DD receiver as well as the penalty $(\Delta)$ between them.

\begin{tabular}{cccc}
\hline \hline \multirow{2}{*}{$\begin{array}{c}\text { Mod. } \\
\text { Scheme }\end{array}$} & \multicolumn{2}{c}{ Sensitivity $(\mathrm{dBm})$} & \multirow{2}{*}{$\Delta(\mathrm{dB})$} \\
\cline { 2 - 3 } & Unclipping & $\begin{array}{c}\text { Hard } \\
\text { Clipping+filtering }\end{array}$ & \\
\hline OFDM & -14.4 & -12.1 & 2.3 \\
FBMC & -14.2 & -11.8 & 2.4 \\
UFMC & -14.4 & -12.0 & 2.4 \\
GFDM & -13.7 & -11.1 & 2.6 \\
\hline \hline
\end{tabular}

based on a previous one, and uses a DFB as LO, see Fig. 2. In particular, the bit error rate (BER) vs. received optical power $\left(P_{R X}\right)$ has been evaluated for back-to-back $(B 2 B)$ and $25 \mathrm{~km}$ of SSMF as shown in Fig. 6 and Fig. 7. The BER limit of $2.2 \cdot 10^{-}$ ${ }^{3}$, corresponding to $7 \%$ overhead $(\mathrm{OH})$ for forward error corrections (FEC) has been considered [30]. The sensitivity is then defined as the minimum received power for reaching that BER limit.

Table I summarises the sensitivities depicted in Fig. 6 of unclipped and hard clipped-and-filtered OFDM, FBMC, UFMC and GFDM signals for the DD receiver. From Table I, it can be observed that unclipped OFDM, FBMC and UFMC have similar sensitivities, around $-14.3 \mathrm{dBm}$, while the GFDM sensitivity is $\sim 0.6 \mathrm{~dB}$ worse. That sensitivity degradation is due to the loss of orthogonality between sub-carriers, owing to the pulse shaping used at the transmitter. From Table I, it can also be observed that the penalty in the sensitivity when the signals are hard clipped to $50 \%$ of the peak is around $2.4 \mathrm{~dB}$. That penalty in the sensitivity is due to the in-band noise caused by the clipping. Negligible transmission penalties are observed on the obtained sensitivities shown in Fig. 6 when the optical signal is launched into $25 \mathrm{~km}$ of SSMF.

Figure 7 shows $\sim 12.2 \mathrm{~dB}$ enhancement in the sensitivity of the considered transmission system when the DD receiver is upgraded to a QCD receiver with a DFB, biased to provide $+14.3 \mathrm{dBm}$ of output power, as LO. That system enhancement has been measured when signals are clipped and filtered. Similar improvement in the sensitivity can be obtained for unclipped signals. New B2B sensitivities are outlined in Table 


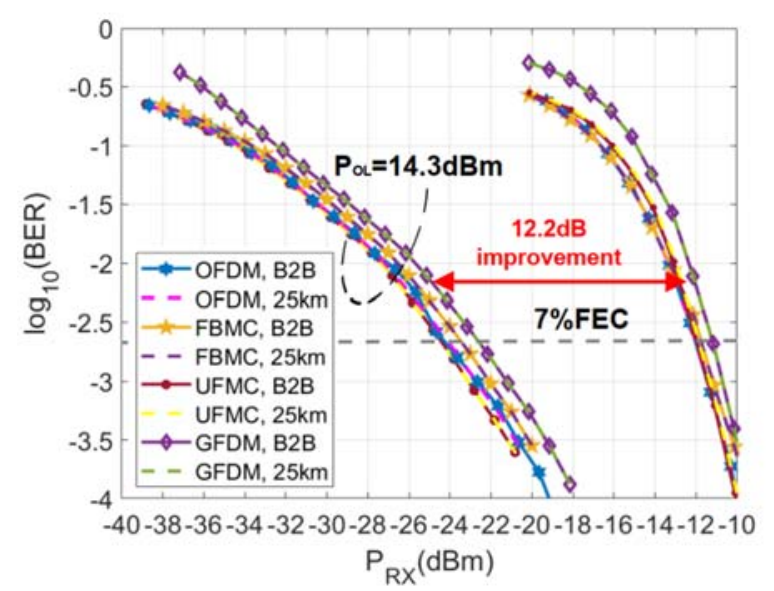

Fig. 7: BER vs. received optical power $\left(\mathrm{P}_{\mathrm{RX}}\right)$ comparison of clipped-andfiltered OFDM, UFMC, GFDM and FBMC signals for the DD and QCD receivers.

TABLE II

Summary of B2B sensitivities of unclipped and clipped-and-filtered OFDM, FBMC, UFMC and GFDM signals for QCD receiver.

\begin{tabular}{ccc}
\hline \hline \multirow{2}{*}{ Mod. Scheme } & \multicolumn{2}{c}{ Sensitivity $(\mathrm{dBm})$} \\
\cline { 2 - 3 } & Unclipping & Hard Clipping+filtering \\
\hline OFDM & -26.8 & -24.5 \\
FBMC & -25.9 & -23.5 \\
UFMC & -27.2 & -24.8 \\
GFDM & -25.4 & -22.8 \\
\hline \hline
\end{tabular}

2. From Fig. 7, it can be observed that those sensitivities do not change when $25 \mathrm{~km}$ SSMF is used. Finally, it has also been experimentally checked that the evolution of the sensitivity of the QCD versus the LO power follows a similar behaviour of the QCD receiver analysed in [26].

\section{Crosstalk interference measure}

As can be observed in Fig. 5, different multicarrier waveforms present different OOB noise levels. For a fronthaul network scenario, as proposed in this paper, see Fig. 1, in which each DWDM channel is divided into two sub-channels, one for upstream and the other for downstream, the OOB noise determines the minimal frequency grid required between different users' spectral demands. On the other hand, Fig. 5 also shows that the OOB noise depends on if the clipping+filtering technique is used or not to reduce the PAPR of transmitted signals. Thus, the OOB noise is maximum when clipping+filtering technique is used and, minimum when not. Therefore, the worst case to determine the required grid is determined by the clipping+filtering technique.

Fig. 8 shows the BER penalty for clipped-and-filtered OFDM/FBMC/UFMC/ GFDM, when the frequency difference $(\Delta f)$ between two adjacent channels, with similar features, is modified. That BER penalty has been measured using the QCD receiver, defining $\Delta f$ as the electrical frequency separation between the central carrier of the measured channel and the interfering channel. For this measurement, the second transmitter has been enabled in the setup proposed in Fig. 2, so as to generate the interfering channel, whose wavelength has

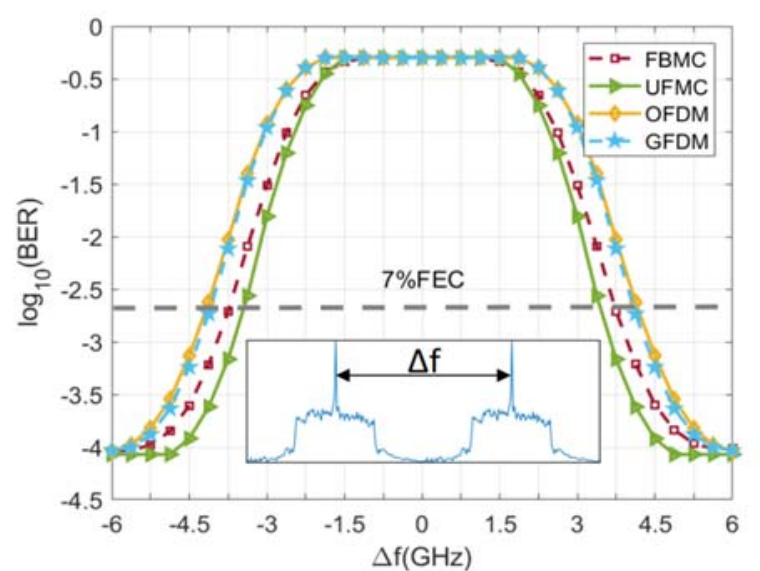

Fig. 8: BER vs. electrical frequency span $(\Delta \mathrm{f})$ between two adjacent channels of the same type: OFDM, UFMC, GFDM and FBMC. Inlet: Electrical frequency span definition between two adjacent channels.

been tuned in steps of $0.75 \mathrm{GHz}$ for a maximum spectral span of $\pm 6 \mathrm{GHz}$. Eventually, to reduce as much as possible the interference of the adjacent channel, a digital 128-coefficient band-pass FIR filter has been considered for the IF at the receiver side (see Fig. 2). From Fig. 8, it can be observed that for an FEC limit of $7 \% \mathrm{OH}$, the required $\Delta f$ for OFDM and GFDM are very similar, $\sim 4.125 \mathrm{GHz}$. For that same FEC limit, the required $\Delta f$ for $\mathrm{FBMC}$ and UFMC are $\sim 3.750 \mathrm{GHz}$ and $\sim 3.375 \mathrm{GHz}$, respectively. These results confirm that UFMC, with $18.2 \%$ of the required frequency difference less than OFDM, provides the best spectral localisation, followed by FBMC, with a reduction of $9.1 \%$. In the case of GFDM, no improvement has been obtained with regard to OFDM due to the fact that GFDM's OOB noise level is slightly lower compared to OFDM, see Fig. 5(e). For the obtained required frequency difference, the penalty over the sensitivity of the considered receiver gets worse in just $1 \mathrm{~dB}$ for all measured multicarrier waveforms.

\section{D.Discussion: optical splitting ratio}

High energy efficiency, low power consumption, and high throughput performance are the main motivations behind mobile network architectures and technological evolution. In the context of incoming C-RANs, in which massive small cells have to be deployed, high-layer split fronthaul will play an important role. In general, the optical splitting ratio of the physical layer of a network depends on the available power budget $\left(P_{B}\right)$ according to the technology used. Thus, the

TABLE III

Summary of available power budget $\left(P_{B}\right)$ of unclipped and clippedand-filtered OFDM, FBMC, UFMC and GFDM signals for direct detection (DD) and quasicoherent detection (QCD) receiver.

\begin{tabular}{ccccc}
\hline \hline & \multicolumn{4}{c}{$P_{B}(\mathrm{~dB})$} \\
\cline { 2 - 5 } $\begin{array}{c}\text { Mod. } \\
\text { Scheme }\end{array}$ & Unclipped & $\begin{array}{c}\text { Clipped- } \\
\text { and- } \\
\text { filtered }\end{array}$ & Unclipped & $\begin{array}{c}\text { Clipped- } \\
\text { and- } \\
\text { filtered }\end{array}$ \\
\cline { 2 - 5 } & 8.4 & 6.1 & 21.8 & 19.5 \\
OFDM & 8.2 & 5.8 & 20.9 & 18.5 \\
FBMC & 8.4 & 6.0 & 22.2 & 19.8 \\
UFMC & 7.7 & 5.1 & 20.4 & 17.8 \\
GFDM & &
\end{tabular}


sensitivity of the optical receiver used for the RRH implementation is an important parameter to determine the available $\mathrm{P}_{\mathrm{B}}$. Based on previous analysis, beside the type of receiver used, a DD or QCD receiver, the sensitivity also depends on if a clipping technique is used to improve the energy efficiency or not, the presence of adjacent channels, and, to a lesser extent, the multicarrier modulation scheme. Table III shows the available $P_{B}$ for OFDM, FBMC, UFMC and GFDM for both $\mathrm{DD}$ and $\mathrm{QCD}$ receivers when signals are unclipped and, clipped and filtered. These values have been calculated bearing in mind that the optical power at the central office's output is $0 \mathrm{dBm}$. Different users' spectral demands are allocated into the same DWDM channel, with the minimal spectral separation calculated in Section III.C, for a penalty of $1 \mathrm{~dB}$, $25 \mathrm{~km}$ of SSMF, and the sensitivity values shown in Tables I and II. Then, the available $P_{B}$ values, shown in Table III, are supposed to be consumed by aWDM multiplexer and a power splitter, see Fig. 1. The main idea of using a power splitter is to share the same DWDM channel between different antennas. Typically, the insertion loss of an arrayed waveguide grating (AWG)-based WDM multiplexer that works for all C-band (4THz of bandwidth) is of $5 \mathrm{~dB}$ for DWDM channel bandwidths of $100(200) \mathrm{GHz}$. Therefore, the total optical splitting ratio (TOSR) reachable when RRHs are based on DD receivers, is of 40(20), while the TOSR value when QCD receivers are used is of 640(320). That means that, for QCD-receiver-based RRH, the TOSR is 16 times higher than DD-receiver-based RRH. As can be expected, these TNSR do not depend on either the modulation scheme used or if a clipping technique is applied over the transmitted signals.

\section{IV.CONCLUSIONS}

In this paper, new $5 \mathrm{G}$ multicarrier waveforms (FBMC, UFMC and GFDM) and OFDM have been experimentally assessed for high-layer split DWDM-PON-based Fronthaul with RoF technology. In particular, spectral efficiency, peak-toaverage power ratio, power spectral density and, the sensitivity for a direct-detection receiver and for a quasi-coherentdetection receiver have been studied. In order to increase the energy efficiency of the communication system, the effects of the use of a hard clipping technique over transmitted signals has also been experimentally assessed. Additionally, for u-DWDM application, the frequency difference between two similar adjacent channels with a $1 \mathrm{~dB}$-penalty has been measured.

Two main conclusions can be extracted from this detailed experimental assessment. On the one hand, it has been demonstrated that good performances can be achieved with hard clipping in spectral conformed, considered modulation formats: OFDM and new $5 \mathrm{G}$ multicarrier waveforms (FBMC, UFMC and GFDM). In particular, results show that: (i) GFDM and FBMC present a slightly higher spectral efficiency $(16 \%)$ than OFDM and UFMC, (ii) hard clipping ( $50 \%$ of the peak of the transmitted symbol) provides $4 \mathrm{~dB}$ PAPR reduction, at the cost of $2.3 \mathrm{~dB}$ (ODFM) to $2.6 \mathrm{~dB}$ (GFDM) sensitivity penalty, after the appropriated filtering of the transmitted signal, to reduce the out-of-band noise, and (iii) the respectively directdetection and quasi-coherent sensitivities are around $-14 \mathrm{dBm}$ and $-24.2 \mathrm{dBm}$. On the other hand, UFMC and FBMC have been identified as the most appropriated $5 \mathrm{G}$ modulation formats for
u-DWDM-PON. The UFMC shows the best spectral localisation followed by FBMC, which reduce by $18.2 \%$ and $9.1 \%$, respectively, the required frequency differences between two adjacent channels regarding OFDM, while GFDM does not provide improvement. Finally, while new 5G multicarrier waveforms (FBMC, UFMC and GFDM) provide similar sensitivities as the previous OFDM, and, for example, considering 100(200)GHz-bandwidth DWDM channels, the total network splitting ratio is $640(320)$ for quasi-coherentdetection receivers, UFMC provides an increase of the network's capacity close to $20 \%$, potentially delivering up to $29(59) 2 \mathrm{GHz}$ channels with 290(590)Gbps per DWDM channel, whereas CPRI would transmit 2(4) $100 \mathrm{MHz}$ channels in that same optical bandwidth.

\section{REFERENCES}

[1] M. Fiorani et al., "Challenges for 5G transport networks," in Proc. ANTS 2014, 2014.

[2] T. Pfeiffer, "Next generation mobile fronthaul and midhaul architectures," IEEE J. Opt. Commun. Netw., vol. 7, no. 11, pp. B38-B45, 2015.

[3] P. Rost et al., "Cloud technologies for flexible 5G radio access networks," IEEE Commun. Mag., vol. 52, no. 5, pp. 68-76, 2014.

[4] Ericsson, "Ericsson mobility report: On the pulse of the networked society," Ericsson, Stockholm, Sweden, Tech. Rep. EAB-16:018498, 2016.

[5] Cisco, "Cisco visual networking index: Global mobile data traffic forecast update, 2016c2021 white paper," Cisco, Jialefuniya, America, Tech. Rep. Cisco white paper, 2017.

[6] A. Pizzinat, P. Chanclou, T. Diallo, and F. Saliou, "Things You Should Know About Fronthaul", in Proc. European Conf. on Optical Communication, Cannes, France, paper Tu.4.2.1, 2014.

[7] I. A. Alimi, A. L. Teixeira and P. P. Monteiro, "Toward an Efficient CRAN Optical Fronthaul for the Future Networks: A Tutorial on Technologies, Requirements, Challenges, and Solutions," in IEEE Communications Surveys \& Tutorials, vol. 20, no. 1, pp. 708-769, Firstquarter 2018.

[8] CPRI Specification V6.1, "Common Public Radio Interface (CPRI)Interface Specification," 2014. Available at http://www.cpri.info/downloads/CPRI v $6 \quad 1$ 2014-07-01.pdf

[9] T. Pfeiffer, "Next generation mobile fronthaul architectures," 2015 Optical Fiber Communications Conference and Exhibition (OFC), Los Angeles, CA, 2015, pp. 1-3.

[10] K. Tanaka and A. Agata, "Next-generation optical access networks for CRAN," 2015 Optical Fiber Communications Conference and Exhibition (OFC), Los Angeles, CA, 2015, pp. 1-3.

[11] Seung-Hyun Cho, Heuk Park, Hwan Seok Chung, Kyeong Hwan Doo, Sangsoo Lee and Jong Hyun Lee, "Cost-effective next generation mobile fronthaul architecture with multi-IF carrier transmission scheme," OFC 2014, San Francisco, CA, 2014, pp. 1-3.

[12] Byung Gon Kim, S. H. Bae, H. Kim and Y. C. Chung, "Optical fronthaul technologies for next-generation mobile communications," 2016 18th International Conference on Transparent Optical Networks (ICTON), Trento, 2016, pp. 1-3.

[13] J. Zhang et al., "Full-duplex quasi-gapless carrier aggregation using FBMC in centralized radio-over-fiber heterogeneous networks" J. Lightwave Technol. 35, 989-996 (2017).

[14] B. G. Kim, H. Kim, and Y. C. Chung, "Impact of multipath interference in the performance of RoF-based mobile fronthaul network implemented by using DML," J. Lightwave technol. 35, 145-151 (2017).

[15] H. Kim, "RoF-based Optical Fronthaul Technology for 5G and Beyond," 2018 Optical Fiber Communications Conference and Exposition (OFC), San Diego, CA, 2018, pp. 1-3.

[16] "10-gigabit-capable symmetric passive optical network (XGS-PON)," ITU-T G.9807 Series of Recommendations, 2016.

[17] H. Rohde, et al., "Coherent Ultra Dense WDM technology for Next Generation Optical Metro and access Networks," J. Lightw. Technol., vol. 32, no. 10, pp. 2041-2052, 2014. 
[18] Jun-ichi Kani, Jun Terada, Ken-Ichi Suzuki, and Akihiro Otaka, "Solutions for Future Mobile Fronthaul and Access-Network Convergence," J. Lightwave Technol. 35, 527-534 (2017).

[19] P. Chanclou, L. Anet Neto, K. Grzybowski, Z. Tayq, F. Saliou, and N. Genay, "Mobile Fronthaul Architecture and Technologies: A RAN Equipment Assessment [Invited]," J. Opt. Commun. Netw. 10, A1-A7 (2018).

[20] H. Sampath, S. Talwar, J. Tellado, V. Erceg, and A. Paulraj, "A fourth-generation MIMO-OFDM broadband wireless system: Design, performance, and field trial results," IEEE Commun. Mag., vol. 40, no. 9, pp. 143-149, Sep. 2002

[21] Y. G. Li and G. Stuber, OFDM for Wireless Communications. Boston, MA, USA: Springer, 2006.

[22] G. L. Stuber et al., "Broadband MIMO-OFDM wireless communications," Proc. IEEE, vol. 92, no. 2, pp. 271-294, Feb. 2004.

[23] Y. Cai, Z. Qin, F. Cui, G. Y. Li and J. A. McCann, "Modulation and Multiple Access for 5G Networks," in IEEE Communications Surveys \& Tutorials, vol. 20, no. 1, pp. 629-646, Firstquarter 2018.

[24] F. Barrami, Y. Le Guennec, E. Novakov, J. Duchamp and P. Busson, “A novel FFT/IFFT size efficient technique to generate real time optical OFDM signals compatible with IM/DD systems," 2013 European Microwave Conference, Nuremberg, 2013, pp. 1247-1250.

[25] B. Glance, "Polarization independent coherent optical receiver," J. Lightwave Technol. 5(2), 274-276 (1987).

[26] J. A. Altabas et al., "Real-time 10Gbps Polarization Independent Quasicoherent Receiver for NG-PON2 Access Networks," 2018 Optical Fiber Communications Conference and Exposition (OFC), San Diego, CA, 2018, pp. 1-3.

[27] M. Bellanger, "Physical layer for future broadband radio systems," 2010 IEEE Radio and Wireless Symposium (RWS), New Orleans, LA, 2010, pp. 436-439.

[28] Gerzaguet, et al., "The 5G candidate waveform race: a comparison of complexity and performance," EURASIP Journal on Wireless Communications and Networking, 2017(1), 13.

[29] Seung Hee Han and Jae Hong Lee, "An overview of peak-to-average power ratio reduction techniques for multicarrier transmission," in IEEE Wireless Communications, vol. 12, no. 2, pp. 56-65, April 2005.

[30] "Forward error correction for high bit-rate DWDM submarine systems," ITU-T Recommendation G.975.1, 2004. 